\title{
Identification of Tsr and Tar Chemoreceptor Arrays in E. coli Inner Membranes
}

\author{
Rosemary S. McAndrew ${ }^{*, * * *}$, E. Ann Ellis ${ }^{* *}$, RunZhi Lai ${ }^{*}$, Michael Manson ${ }^{*, * * *}$, and Andreas \\ Holzenburg, ${ }^{* * *, * * *}$
}

*Department of Biology, ${ }^{* *}$ Microscopy and Imaging Center (MS 2257), and ${ }^{* * *}$ Department of Biochemistry and Biophysics, Texas A\&M University, College Station, TX 77843-2257

Bacterial chemotaxis directs the movement of cells in attractant and repellent chemical gradients. This behavior is regulated by several different transmembrane chemoreceptors that are grouped in patches at the cell pole. The receptors function as self-associating homodimers that span the inner membrane and bind ligands in the periplasmic space ${ }^{1}$. Furthermore, these dimers assemble into rather stable trimers. Receptor-mediated control of flagellar motors occurs through a twocomponent signaling pathway that couples the activity of the histidine protein kinase CheA to receptors through the linker protein $\mathrm{CheW}^{2,3}$. The high sensitivity and broad dose response of chemotaxis is consistent with accumulating evidence from theoretical modeling, structural, and kinetic studies that indicates that the receptors act in larger assemblies ${ }^{4}$. Although no direct evidence for such higher-order interactions has been obtained, a clear understanding of network architecture is integral to elucidating the molecular mechanisms that govern receptor signaling. Previously, TEM analysis was used to investigate structural characteristics of serine (Tsr) receptor arrays relative to their functional roles in vivo by examining their organization in native membranes of $E$. coli in the presence and absence of $\mathrm{CheA}, \mathrm{CheW}$, and the attractant ligand serine ${ }^{5}$. This study expands the investigation to include both $\mathrm{Tsr}$ and aspartate (Tar) receptors aimed at a more comprehensive understanding of receptor networks in vivo.

Receptors were expressed in E. coli strain RP3098, which lacks all chemotaxis proteins, and recovered in inner membranes fractionated on sucrose gradients ${ }^{2}$. Immunolabeling of Tsr or Tar in thin cell sections and membrane preparations, and assays for receptor-coupled CheA kinase activity, were performed to confirm Tsr or Tar expression and the formation of active ternary complexes with CheW and $\mathrm{CheA}^{5,6}$. Samples were deposited on glow-discharged, carbon-coated grids and negatively stained with $2 \%$ aqueous uranyl acetate. Alternately, samples were deposited on grids for immunolabeling of Tsr or Tar in membrane suspensions with receptor-specific polyclonal rabbit primary antibodies and visualization with donkey anti-rabbit IgG conjugated to $6 \mathrm{~nm}$ gold (Jackson Immuno Research, West Grove, PA) and negatively stained with $2 \%$ phosphotungstic acid, $\mathrm{pH} 7.5$.

Both Tsr and Tar receptors appear in membranes as mosaic clusters. Specific gold labeling of Tsr and Tar (Fig. 1 and 2) and assays for receptor-coupled CheA kinase activity confirms that functional receptor assemblies are associated with these clusters. Although coexpression of Tsr and Tar in the same cells results in a 2 to 5 -fold increase in kinase stimulation over that achieved by homogenous receptor populations, supporting synergism, the proportion and distribution of each receptor within presumed mixed clusters from such cells is unknown and currently under investigation. These results provide new evidence that chemoreceptors form organized assemblies and networks to provide heightened control of bacterial motility. Determining the higher-level organization of chemoreceptors in cell membranes is integral to understanding the molecular basis of signaling by wild type complexes. These results will advance our understanding of the functional relationship between chemoreceptor achitecture and highly coordinated and integrated chemotactic behavior. 


\section{References}

[1] J. J. Falke, Proc. Natl. Acad. Sci. USA 99 (2002) 6530.

[2] J. A. Gegner et al., Cell 70 (1992) 975.

[3] S. C. Schuster et al., Nature 365 (1993) 343.

[4] C. A. Studdert and J. S. Parkinson, Proc. Natl. Acad. Sci. USA 101 (2004) 2117.

[5] R. S. McAndrew et al., Microsc. Microanal. 10 (2004) 416.

[6] K. A. Borchovich and M. I. Simon, Cell 63 (1990) 1339.

[7] This research was supported by NIH Grant RO1 GM39736-13 and the Program for Membrane Protein Structure and Function established by Texas A\&M University.
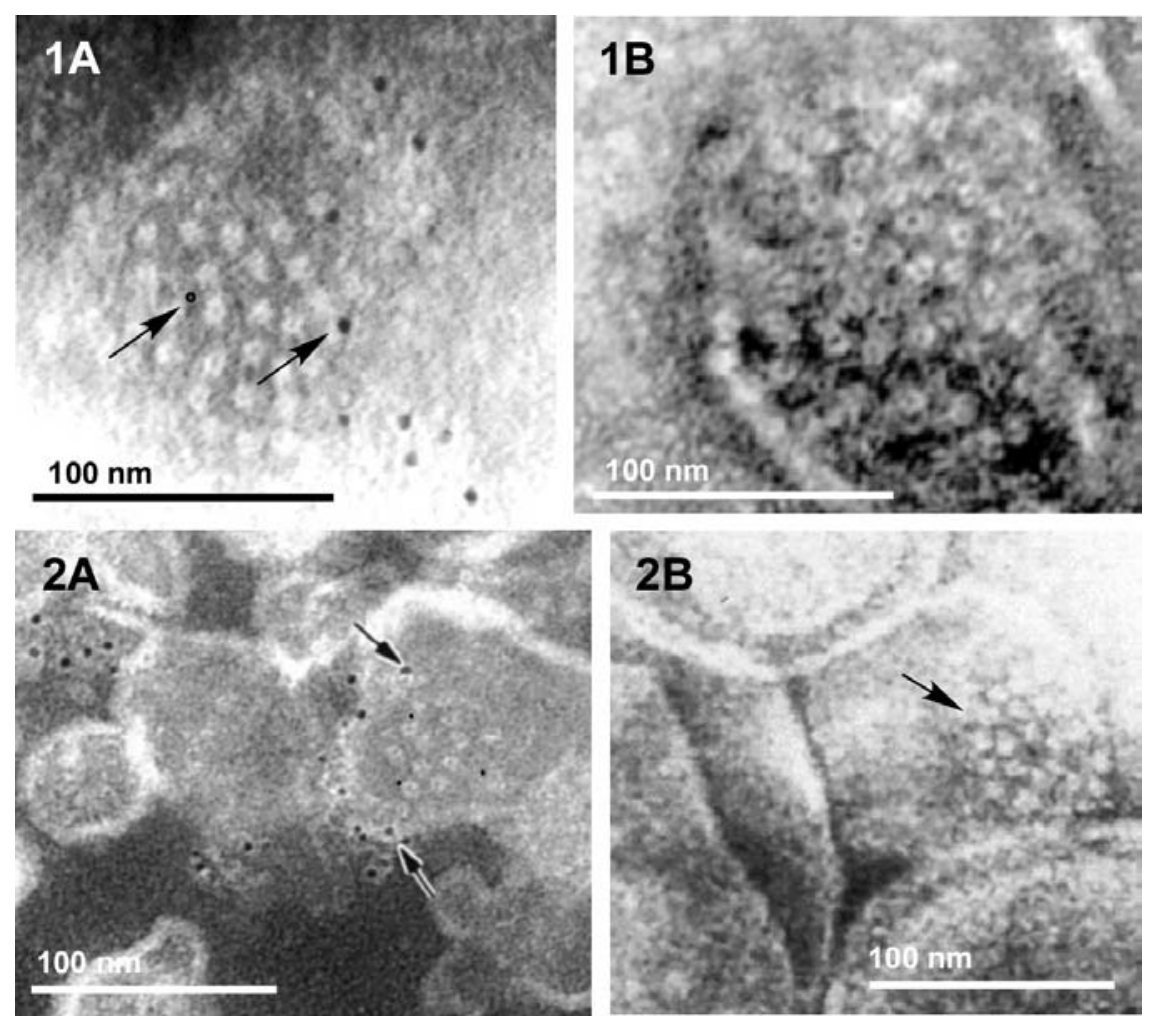

FIG. 1. Images recorded from inner membranes isolated from Tsr-expressing E. coli cells showing (A) Tsr-specific gold labeling (arrows) associated with mosaic clusters or (B) the absence of labeling in clusters from the same membranes processed with anti-Tsr primary antibodies that were preincubated with Tsr prior to incubation with membranes and secondary IgG gold conjugates.

FIG. 2. Images recorded from inner membranes isolated from Tar-expressing E. coli cells showing (A) Tar-specific gold labeling (arrows) associated with mosaic clusters or (B) the absence of labeling in clusters (arrow) from the same membranes processed with IgG gold conjugates only. 